\title{
Plasma Chemokine C-C Motif Ligand 2 as a Potential Biomarker for Prostate Cancer
}

\author{
Martin Lund (D)', Torben B Pedersen (D)', Søren Feddersen ${ }^{2,3}$, Louise D Østergaard $\mathbb{D}^{1,3}$, \\ Charlotte A Poulsen', Christian Enggaard ${ }^{2}$, Mads HA Poulsen $\mathbb{1}^{1,3}$, Lars Lund ${ }^{1,3}$ \\ 'Department of Urology, Odense University Hospital, Odense, Denmark; ${ }^{2}$ Department of Clinical Biochemistry and Pharmacology, Odense \\ University Hospital, Odense, Denmark; ${ }^{3}$ Department of Clinical Research, University of Southern Denmark, Odense, Denmark
}

Correspondence: Lars Lund, Department of Urology, Odense University Hospital, Sdr. Boulevard 29, Odense, 5000, Denmark, Tel +45 5I40 8982 , Fax +45 654I 1726, Email Lars.Lund@rsyd.dk

Purpose: Serum levels of the polypeptide chemokine C-C motif ligand 2 (CCL2) have previously shown potential as a prostate cancer diagnostic and prognostic biomarker. Plasma CCL2 levels may be superior to serum levels as a biomarker because of their potentially lower signal-to-noise ratio.

Materials and Methods: Before initiating a large comparative study of plasma and serum CCL2 levels, we performed a prospective, diagnostic pilot study Of 133 individuals from a clinically relevant population. CCL2 plasma levels were measured using a validated assay kit. Plasma was obtained independently of digital rectal examination.

Results: In this pilot study, we found no relationship between CCL2 plasma values and risk of proven prostate cancer, whereas previous studies found a strong diagnostic relationship between CCL2 serum values and prostate cancer.

Conclusion: Our contribution to the existing literature strengthens the idea that early in the pathological process, CCL2 mainly circulates in large, membrane-enclosed compartments, whereas plasma CCL2 levels increase markedly during disease progression. We conclude that whereas plasma CCL2 levels are not useful as a diagnostic measure, a ratio of CCL2 plasma to serum levels may prove useful as a marker of disease progression, which warrants further study.

Keywords: diagnostics, liquid biopsy, CCL2, monocyte chemoattractant protein 1, MCP1

\section{Introduction}

Prostate cancer $(\mathrm{PCa})$ is one of the more common cancers affecting men. Recent years have brought an increased number of therapeutic options and more accurate diagnostic pathways. Thus, PCa today accounts for less than $10 \%$ of total cancer mortality in males living in the developed world. Additionally, more than $90 \%$ of new PCa cases can be expected to be diagnosed at local or regional stages, for which the 5-year relative survival approaches $100 \% .{ }^{1}$ However, PCa disease progression leading to castration-resistance and eventually metastatic disease is still the cause of reduced quality of life and increased risk of death in a significant number of patients. ${ }^{2}$

A whole series of cytokines and chemokines are necessary for the development of aggressive PCa and subsequent metastasis and may therefore potentially be employed as biomarkers. However, whereas many cytokines have been tested as potential diagnostic or prognostic makers, few have been validated successfully in multiple clinical settings. ${ }^{3-5}$

Increased glandular levels of chemokine (CC motif) ligand 2 (CCL2), also known as monocyte chemoattractant protein 1 (MCP-1), correlate strongly with a negative prognosis. Increased CCL2 presence protects PCa cells from proapoptotic signalling and leads to expression of specific integrins, which enables cancer cell migration and attachment at metastatic sites, especially in bone. ${ }^{6-16}$

Circulating CCL2 serum levels were first found to have a diagnostic potential in a pilot study $^{5}$ comparing serum levels of various cytokines from 15 healthy volunteers to those of 39 PCa patients undergoing radical prostatectomy. A study design comparing healthy volunteers to severely ill patients is very useful for biomarker discovery as the signal 
strength differential is so strong that relatively few participants are required. However, any potential biomarkers must be validated in a clinically relevant setting. The first validation study was performed by a separate group who found that CCL2 serum levels had a diagnostic and prognostic capacity in a cohort of 379 men undergoing transrectal biopsy of the prostate due to suspicion of cancer. ${ }^{15}$

A more recent study indicates that serum CCL2 values predict the outcome of PCa biopsies of patients under active surveillance. ${ }^{17}$ In addition to plasma components, serum contains platelets, white blood cells, vesicles and potentially circulating tumour-cells (CTCs), which may alter the diagnostic usefulness of a specific cytokine. For example, by introducing higher background noise, that mask clinically relevant, low-abundance cytokine biomarkers. ${ }^{18}$ Plasma CCL2 levels could potentially be the preferable matrix for early detection of $\mathrm{PCa}$, which we test in this prospective pilot study.

\section{Materials and Methods}

We consecutively included 142 men referred for transrectal ultrasound-guided prostate biopsy due to suspicion of PCa based on an elevated prostate- specific antigen (PSA) levels or an abnormal digital rectal examination. Among the included patients, nine declined biopsy or their biopsy result was unclear. Plasma was obtained independently of digital rectal examination. Recruitment of patients and storage of sensitive data was performed in accordance with the Declaration of Helsinki. The study was approved by the Southern Regional Ethics Committee, Denmark (Projekt-ID: S-20190036). All participants gave informed consent.

\section{Enzyme-Linked Immunosorbent Assay}

Blood was collected in K2-EDTA tubes and placed on ice. Plasma was isolated by a single centrifugation, performed at $800 \mathrm{~g}$ for 10 minutes at $4^{\circ} \mathrm{C}$. Isolated plasma was frozen at $-80^{\circ} \mathrm{C}$ until analysis.

CCL2 levels were determined using the R\&D Biosystems (UK) Human CCL2/MCP-1 Quantikine ELISA Kit (DCP00) following the manufacturer's instructions. Interassay variation was assessed using a separately obtained pooled plasma sample.

\section{Data Treatment and Statistical Analyses}

$\mathrm{R}$ version 4.1 .2 (2021-11-01) was used to prepare figures and for all statistical analyses. Group data is presented as Tukey box-and-whisker plots. ${ }^{19}$ Kruskal-Wallis' nonparametric test was used to calculate p-values for multiple group comparison. Mann-Whitney nonparametric $U$-test was used for calculating p-values for two- group comparisons. P-values $<0.05$ were considered significant. Area under the curve (AUC) confidence bands for receiver-operating characteristic (ROC) curves were compared using DeLongs's nonparametric approach. The R session log is presented in Supplementary Materials.

\section{Results}

A total of 133 patient samples were available for CCL2 measurement. The median patient age was 68 years (interquartile range: $62-72$ years). Among the 133 patients, 50 had no detectable cancer, 37 had a Gleason score of 6,31 had a score of $7(3+4), 5$ had a score of $7(4+3), 4$ had a score of 8 and 6 had a score of 9-10 (Figure 1). The AUC of CCL2 was 0.521 and incapable of predicting significant cancer (Figure 2). We found no relationship between plasma CCL2 levels and Gleason score or between CCL2 levels and cT stage (Figure 1). Importantly, even though we cannot yet provide an overall survival rate or similar measurement, no observable concentration differences exist across the $\mathrm{PCa}$ and non- $\mathrm{PCa}$ groupings, making it highly unlikely that there is any prognostic capacity in plasma CCL2 levels at the time of diagnosis. The inclusion of 0 within the $95 \%$ confidence interval range is compatible with no diagnostic effect and with the fact that the study is not underpowered; however, repeated independent studies would be required to establish this conclusively. It should be noted that serum CCL2 levels were found to have significant diagnostic abilities with a smaller cohort size. ${ }^{5}$ Sub-analysis of AUC of CCL2 of any cancer type, versus cancer negative biopsies has a comparable outcome (Supplementary Figure 1). 

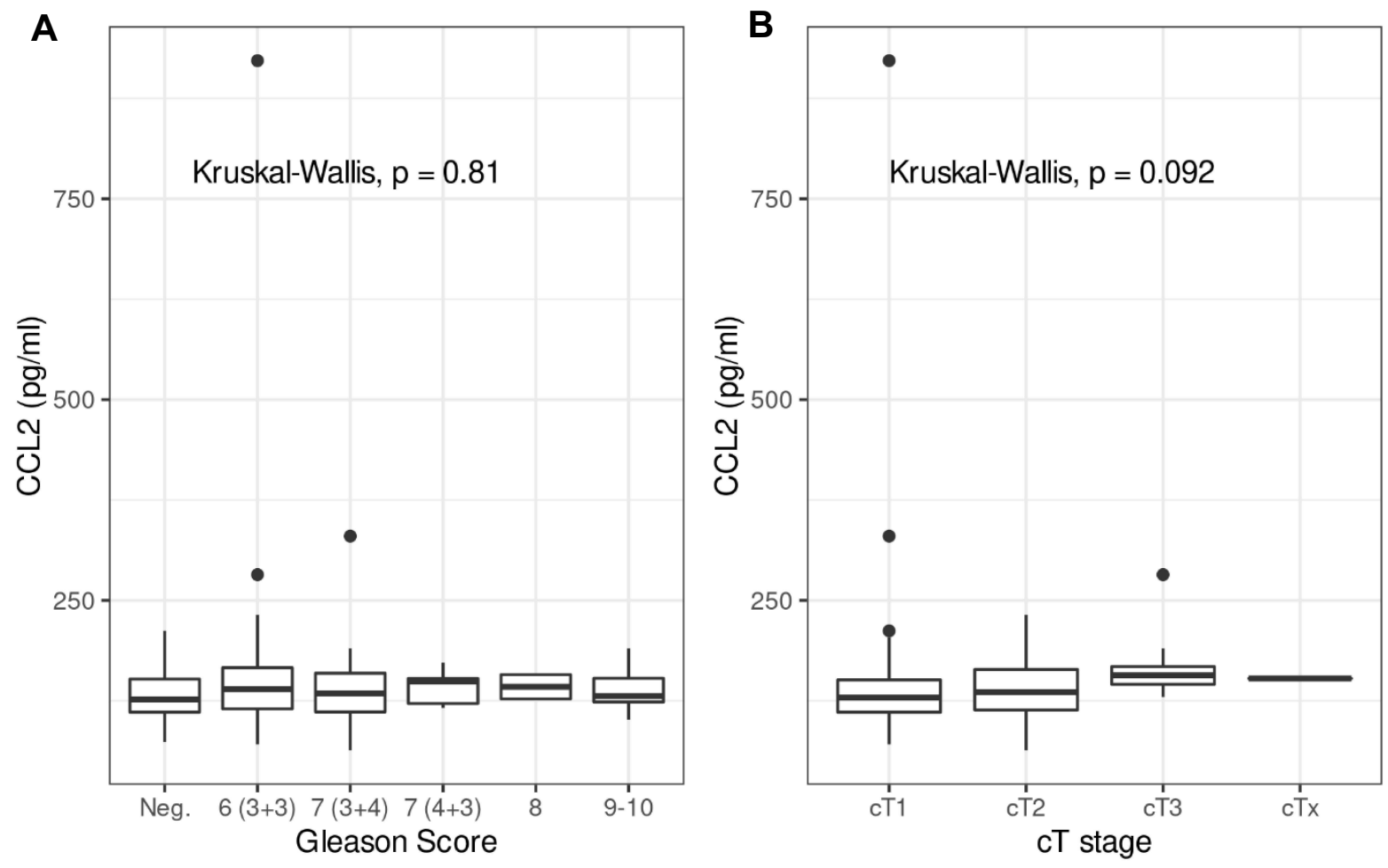

\begin{tabular}{|lccccccc|}
\hline \multicolumn{7}{c|}{ Gleason score } \\
\hline & $\begin{array}{c}\text { Negative } \\
(\mathrm{n}=50)\end{array}$ & $\begin{array}{c}6(3+3) \\
(\mathrm{n}=37)\end{array}$ & $\begin{array}{c}7(3+4) \\
(\mathrm{n}=31)\end{array}$ & $\begin{array}{c}7(4+3) \\
(\mathrm{n}=5)\end{array}$ & $\begin{array}{c}8 \\
(\mathrm{n}=4)\end{array}$ & $\begin{array}{c}9-10 \\
(\mathrm{n}=6)\end{array}$ & P-value \\
\hline Plasma CCL2 $(\mathrm{pg} / \mathrm{ml})$ & & & & & & & \\
Min & 73.8 & 70.8 & 63.4 & 116.2 & 126.6 & 101.6 & \\
Max & 212.0 & 922.0 & 330.0 & 172.6 & 158.6 & 190.2 & \\
Median & 126.5 & 139.4 & 134.0 & 149.0 & 142.4 & 130.9 & 0.813 \\
PSA (ng/ml) & & & & & & & \\
Min & 2.5 & 3.3 & 3.5 & 7.2 & 9.3 & 8.2 & \\
Max & 17.0 & 21.0 & 17.0 & 14.0 & 16.0 & 33 & \\
Median & 6.2 & 7.9 & 7.1 & 12.0 & 11.5 & 17.0 & $2.39 \mathrm{e}-05^{*}$ \\
\hline
\end{tabular}

Figure I Gleason score and T stage. Tukey box-and-whisker plot of EDTA-plasma CCL2 levels (pg/mL) plotted against (A) Gleason score. (B) cT stage. Table shows patient PSA and EDTA-plasma CCL2 levels $(\mathrm{pg} / \mathrm{mL})$ levels ability to predict Gleason score. For comparison of multiple group means Kruskal-Wallis' nonparametric test was used, p-values $<0.05$ were considered significant $(*)$.

\section{Discussion}

Our study indicates that circulating CCL2 participating in the priming of (bone) metastatic sites is associated with white blood cells, CTC or other large circulating element(s) and that freely circulating CCL2 levels do not increase until onset of therapy. 


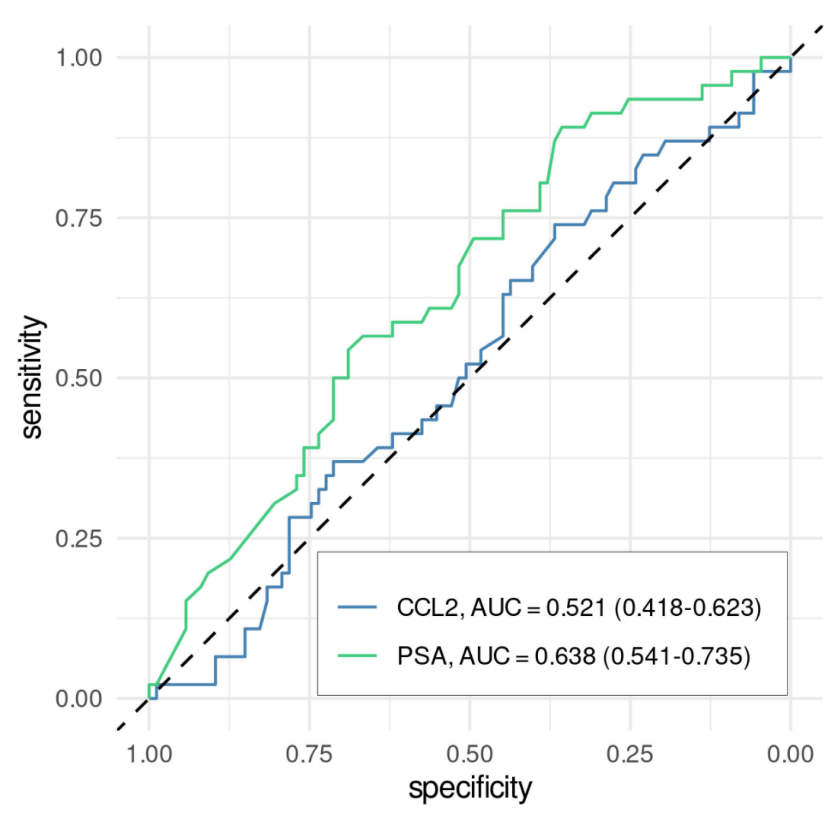

\begin{tabular}{|ccccc|}
\hline & Non-significant & Significant & Median of the & p-value \\
& Cancer $(\mathrm{n}=87)$ & Cancer $(\mathrm{n}=46)$ & Difference 95\% Cl & - \\
Median PSA, $\mathrm{ng} / \mathrm{ml}$ & 7.0 & 8.4 & $1.4[0.3,2.6]$ & $0.00913^{*}$ \\
Median CCL2, $\mathrm{pg} / \mathrm{ml}$ & 130.8 & 133.0 & $2.2[-10.6,14.4]$ & 0.698 \\
\hline
\end{tabular}

Figure 2 CCL2 \& PSA levels ROC and predictive power. PSA and CCL2 receiver operating characteristic curve for significant cancer versus Gleason Score 6 (3+3) or negative biopsies and ability to predict significant cancer. Wilcoxon nonparametric test was used for two-group comparison, $\mathrm{p}$-values $<0.05$ were considered significant $(*)$. Confidence bands for area under the curve (AUC) for receiver operating characteristic (ROC) curves were compared using DeLongs's nonparametric approach.

Serum CCL2 levels has already shown potential as a useful diagnostic marker in both an exploratory pilot study and two clinically relevant settings. We therefore proceeded to test the potential of plasma CCL2 levels in a clinical setting, bypassing an initial comparison of healthy controls as irrelevant because plasma CCL2 levels would at the very least have to be comparable with serum CCL2 levels in terms of diagnostic efficacy in order for it to be relevant to study in this context. It is possible to view this omission as a weakness of this pilot study, but we feel it is justified by previous work on serum CCL2 levels as a potential biomarker.

CCL2 and its receptors have been studied extensively in cell culture and animal models of PCa, showing the ability to act as an androgen-independent, auto- and paracrine anti-apoptotic growth factor and mediator of the metastatic process. ${ }^{6-10}$ A recent small study of multiple end-stage cancers found plasma CCL2 levels to have a prognostic capacity across the different cancers and to increase following radiation therapy, but only four PCa patients were included in a total cohort of 21 patients with cancer of the prostate, colon, kidney or breast. Since the analysis pooled a small cohort of multiple cancer types, this conclusion needs to be interpreted with some caution. ${ }^{20}$ A recent Mendelian randomisation study found that genetically predicted higher plasma levels of CCL2 increased the risk of developing PCa, which supports the concept of using CCL2 as a PCa biomarker. ${ }^{21}$ However, gene variants causing a problematic increase in CCL2 plasma levels may be too rare to warrant the use of plasma CCL2 levels as a diagnostic biomarker. In any case, such variants are better screened for by genetic sequencing, which needs only occur once in an individual's life-time and may be performed decades before development of PCa.

Histological studies have found that PCa tissue and the surrounding inflamed zone can express high levels of CCL2 and that CCL2 has a central role in preparing metastatic sites. ${ }^{6-10,22-24}$ CCL2 must either circulate in some form to facilitate the formation of pre-metastatic sites or, perhaps less likely, be produced separately at potential metastatic sites by some other means. The present study indicates that quantities of CCL2 sufficient to promote disease progression do not initially circulate as a free solute in the blood stream in PCa patients. Combined with previously published work on 
CCL2 levels in serum from PCa patients, the present study indicates that in individuals without genetic variants leading to chronic elevation of plasma CCL2 levels and prior to treatment, CCL2 circulates with some combination of white blood cells, CTCs and extracellular vesicles large enough to enter the buffy coat during plasma preparation. ${ }^{3-5,15,17,21}$ The largest study population of PCa patients versus non-malignant patients found serum values of $224 \mathrm{pg} / \mathrm{mL}$ CCL2 in cancer-free patients versus $246 \mathrm{pg} / \mathrm{mL}$ CCL2 in cancer patients. ${ }^{15}$ We found $130.8 \mathrm{pg} / \mathrm{mL}$ versus $133 \mathrm{pg} / \mathrm{mL}$ in the same categories in plasma, a differential value indicating that roughly $60 \%$ of total CCL2 is carried in the plasma fraction in non-malignant patients compared with $54 \%$ of total CCL2 in PCa patients. Obviously, one should draw no strong conclusions based on comparisons of measurements performed in two separate studies in different biological matrices; however, the finding does suggest that the ratio of CCL2 in plasma/serum may contain important diagnostic and prognostic information, which may potentially be available at a relatively limited extra analysis. Studies comparing serum extracellular vesicles have found an altered composition in serum when comparing benign prostatic hyperplasia, healthy controls and PCa patients; this finding further strengthens the idea that PCa serum carries biomarkers in vesicular compartments that may improve diagnosis. ${ }^{25}$ It is clear that in terms of diagnostic potential alone, measurement of plasma CCL2 levels is not relevant compared to measurement of serum. Ideally we would have wanted to quantify CCL2 levels in specific tissue biopsies and compare expression levels to both serum and plasma CCL2 levels. Future work on CCL2 as a diagnostic and prognostic biomarker should include this head-to-head comparison.

Several PCa studies have found tumour cell death to be associated with surgical interventions, and castration therapy imposes a selective pressure on tumours that increases in CCL2 expression, which promotes castration resistance and metastasis. ${ }^{6-10,22-24}$ It would be interesting to study CCL2 levels in plasma and serum in the same patient population when they start to undergo therapy as both relative concentrations and the distribution of CCL2 between plasma and serum may carry biomarker potential. This may potentially have several benefits including the potential to improve monitoring of patients undergoing castration therapy, making CCL2 interesting as a potential PCa biomarker. Such testing should ideally measure other candidate biomarkers such as PIWIL2 in order to allow head to head comparison and discover potential biomarker synergies. ${ }^{26}$

\section{Conclusion}

We conclude that unlike serum CCL2, plasma CCL2 is not a potential diagnostic biomarker. We propose that serum and plasma CCL2 levels be measured in patients at diagnosis prior to onset of treatment and at suitable intervals during castration therapy. This would enable concurrent study of prognostic properties and allow each measurement to serve as a disease progression marker and to determine if synergistic effects are associated with testing in both matrices.

\section{Acknowledgments}

We wish to thank the volunteers that donated plasma for this project. This project was partially funded by The Danish Cancer Society.

\section{Disclosure}

The authors declare that they do not have any competing interests.

\section{References}

1. Taitt HE. Global Trends and Prostate Cancer: a Review of Incidence, Detection, and Mortality as Influenced by Race, Ethnicity, and Geographic Location. Am J Mens Health. 2018;12(6):1807-1823. doi:10.1177/1557988318798279

2. Tsuzuki S, Park SH, Eber MR, Peters CM, Shiozawa Y. Skeletal complications in cancer patients with bone metastases. Int J Urol. 2016;23(10):825832.

3. Adekoya TO, Richardson RM. Cytokines and Chemokines as Mediators of Prostate Cancer Metastasis. Int J Mol Sci. 2020;21(12):4449.

4. Gong D, Wang Y, Wang Y, et al. Extensive serum cytokine analysis in patients with prostate cancer. Cytokine. 2020;125:154810.

5. Tsaur I, Noack A, Makarevic J, et al. CCL2 Chemokine as a Potential Biomarker for Prostate Cancer: a Pilot Study. Cancer Res Treat. 2015;47 (2):306-312.

6. Roca H, Varsos Z, Pienta KJ. CCL2 Protects Prostate Cancer PC3 Cells from Autophagic Death via Phosphatidylinositol 3-Kinase/AKT-dependent Survivin Up-regulation. J Biol Chem. 2008;283(36):25057-25073. 
7. Qian DZ, Rademacher BLS, Pittsenbarger J, et al. CCL2 is induced by chemotherapy and protects prostate cancer cells from docetaxel-induced cytotoxicity. Prostate. 2010;70(4):433-442.

8. Loberg RD, Tantivejkul K, Craig M, Neeley CK, Pienta KJ. PAR1-mediated RhoA activation facilitates CCL2-induced chemotaxis in PC-3 cells. $J$ Cell Biochem. 2007;101(5):1292-1300.

9. Lin T-H, Liu -H-H, Tsai T-H, et al. CCL2 increases $\alpha \mathrm{v} \beta 3$ integrin expression and subsequently promotes prostate cancer migration. Biochimica et Biophysica Acta. 2013;1830(10):4917-4927.

10. Tsaur I, Rutz J, Makarević J, et al. CCL2 promotes integrin-mediated adhesion of prostate cancer cells in vitro. World J Urol. 2015;33(7):10511056.

11. Loberg RD, Ying C, Craig M, et al. Targeting CCL2 with systemic delivery of neutralizing antibodies induces prostate cancer tumor regression in vivo. Cancer Res. 2007;67(19):9417-9424.

12. Zhang J, Patel L, Pienta KJ. CC chemokine ligand 2 (CCL2) promotes prostate cancer tumorigenesis and metastasis. Cytokine Growth Factor Rev. 2010;21(1):41-48.

13. Izhak L, Wildbaum G, Weinberg U, et al. Predominant expression of CCL2 at the tumor site of prostate cancer patients directs a selective loss of immunological tolerance to CCL2 that could be amplified in a beneficial manner. J Immunol. 2010;184(2):1092-1101.

14. Pienta KJ, Machiels J-P, Schrijvers D, et al. Phase 2 study of carlumab (CNTO 888), a human monoclonal antibody against CC-chemokine ligand 2 (CCL2), in metastatic castration-resistant prostate cancer. Invest New Drugs. 2013;31(3):760-768.

15. Izumi K, Mizokami A, Lin HP, et al. Serum chemokine (CC motif) ligand 2 level as a diagnostic, predictive, and prognostic biomarker for prostate cancer. Oncotarget. 2016;7(7):8389-8398.

16. Natsagdorj A, Izumi K, Hiratsuka K, et al. CCL2 induces resistance to the antiproliferative effect of cabazitaxel in prostate cancer cells. Cancer Sci. 2019;110(1):279-288.

17. Ahmad AE, Mohammed A, Bhindi B, et al. Serum Adipokines as Predictors for the Outcome of Prostate Biopsies at Early Stage Prostate Cancer Diagnosis. Cancer Manag Res. 2019;11:10043-10050.

18. Rosenberg-Hasson Y, Hansmann L, Liedtke M, Herschmann I, Maecker HT. Effects of serum and plasma matrices on multiplex immunoassays. Immunol Res. 2014;58(2):224-233.

19. Tukey JW. Exploratory data analysis: reading, Mass. 1977.

20. Merlano MC, Abbona A, Paccagnella M, et al. Cytokine Profiling of End Stage Cancer Patients Treated with Immunotherapy. Vaccines. 2021;9 (3):764.

21. Sun X, Ye D, Du L, Qian Y, Jiang X, Mao Y. Genetically predicted levels of circulating cytokines and prostate cancer risk: a Mendelian randomization study. Int $J$ Cancer. 2020;147(9):2469-2478.

22. Lu Y, Cai Z, Xiao G, et al. CCR2 expression correlates with prostate cancer progression. J Cell Biochem. 2007;101(3):676-685.

23. Roca H, Varsos ZS, Sud S, Craig MJ, Ying C, Pienta KJ. CCL2 and Interleukin-6 Promote Survival of Human CD11b+ Peripheral Blood Mononuclear Cells and Induce M2-type Macrophage Polarization. J Biol Chem. 2009;284(49):34342-34354.

24. Tsai Y-C, Chen W-Y, Abou-Kheir W, et al. Androgen deprivation therapy-induced epithelial-mesenchymal transition of prostate cancer through downregulating SPDEF and activating CCL2. Biochim Biophys Acta Mol Basis Dis. 2018;1864(5 Pt A):1717-1727.

25. Salvi S, Bandini E, Carloni S, et al. Detection and Investigation of Extracellular Vesicles in Serum and Urine Supernatant of Prostate Cancer Patients. Diagnostics. 2021;11(3):466.

26. Tosun H, Demirtaş A, Sönmez G, Tombul ŞT, Akalın H, Özkul Y. Can the expression level of PIWIL 2 gene be a serum marker for prostate cancer? A single-center prospective study. Turk J Urol. 2019;45(Supp. 1):S22-s5.

Research and Reports in Urology

\section{Publish your work in this journal}

Research and Reports in Urology is an international, peer-reviewed, open access journal publishing original research, reports, editorials, reviews and commentaries on all aspects of adult and pediatric urology in the clinic and laboratory including the following topics: Pathology, pathophysiology of urological disease; Investigation and treatment of urological disease; Pharmacology of drugs used for the treatment of urological disease. The manuscript management system is completely online and includes a very quick and fair peer-review system, which is all easy to use. Visit http://www.dovepress.com/testimonials.php to read real quotes from published authors.

Submit your manuscript here: https://www.dovepress.com/research-and-reports-in-urology-journal 